\title{
REPEATED PHLEBOTOMY-INDUCED LIVER CELL NECROSIS IN RATS
}

\author{
Toshiharu Sakai, Satoshi Yoshida, Ryuhei Fujimoto, Takanori Hanada, \\ Yasuhiro Sakurai, Aisuke Nii, Hideaki Okamiya, and Hisao Miki \\ Product Development Laboratories, Yamanouchi Pharmaceutical Co., Lid.
}

Yasuo Nomura

Department of Veterinary Pathology, School of Veterinary Medicine. Azabu University

\begin{abstract}
Alstract : Rats deprived of blood, amounting to about one percent of body weight, via orbital venous plexus phlebotomy for 2 weeks developed severe anemia. Their mean hematocrit value was 19\%, and centrolobular liver cell necrosis was observed in animals with hematocrit value of $20 \%$ or less at the end of the study. It is obvious that the animals must have been in a severe anemic condition to develop liver cell necrosis. Thus, anemia should be considered as a causative factor of liver cell necrosis in toxicity studies in which the animals are in severe anemia attributable to drug administration. (J Toxicol Pathol 2: 175 179, 1989)
\end{abstract}

Key words: Anemia, Liver cell necrosis, Rat

\section{Introduction}

Liver cell necrosis is one of the changes encountered in toxicity studies of chemicals, and anemia- or ischemia- induced liver cell necrosis has also been reported by some researchers ${ }^{1-10}$. However, it is difficult to distinguish the true cause of liver cell necrosis in animals associated with anemia, because of few data on the role of anemia in provoking liver cell necrosis.

This study was performed to examine the role and degree of anemia on the occurrence of liver cell necrosis in rats routinely used in toxicity studies.

\section{Materials and Methods}

Animals

Fourty-three male Fischer 344 and SD rats purchased from Charles River Japan Co. Ltd.

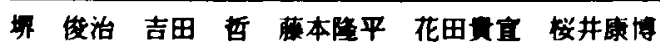

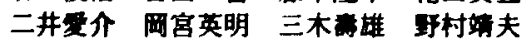

Accepted for publication: June 1, 1989

Mailing address: Toshiharu Sakai, Product Development Laboratories, Yamanouchi Pharmaceutical Co., Ltd., Azusawa 1-1-8, Itabashi, Tokyo 174, Japan.
(Kanagawa pref.), 7 to 11 weeks of age at the start of the study, were used.

The animals were housed individually in suspended stainless steel cages. They were given commercial pellets, CRF-I (Charles River Japan Co. Ltd.), and tap water at libitum. The room was set at $23 \pm 2 . \mathrm{C}$ of temperature and $55 \pm 10 \%$ of humidity, and had an artificial light for 13 hours a day.

\section{Depletion of blood}

Blood amounting to about one percent of the body weight was removed from the orbital venous plexus under light ether anesthesia almost every day for 2 weeks.

\section{Hematology and plasma biochemistry}

Hematological tests were performed once or twice a week for erythrocyte counts, hematocrit (HCT) values and reticulocyte counts, and plasma biochemical tests for glutamic-oxaloacetic transaminase (GOT), glutamic-pyruvic transaminase (GPT), and alkaline phosphatase (ALP) using the blood collected by phlebotomy.

Histopathological examinations

On day 15 of the experiment, all animals were sacrificed by exsanguination under ether anesthsia. 
The liver was dissected, fixed in 10\% phosphatebuffered formalin, embedded in paraffin, sectioned and stained with hematoxylin and eosin (H.E.), periodic acid-Schiff (PAS) or trichrome stain for histopathological examination.

Histopathological findings of the liver were divided into three groups on grade basis as follows: Grade 0 ; normal, Grade 1 ; slight hepatocellular degeneration/necrosis and Grade 2 ; moderate to severe hepatocellular degeneration/ne- crosis.

\section{Results}

Mean HCT values, erythrocyte and reticulocyte counts in rats throughout the experimental period are shown in Fig. 1. HCT values decreased rapidly in the first week and gradually thereafter. At necropsy, HCT values were under $20 \%$ in half of the animals. The half of the animals showed severe anemia with HCT value of

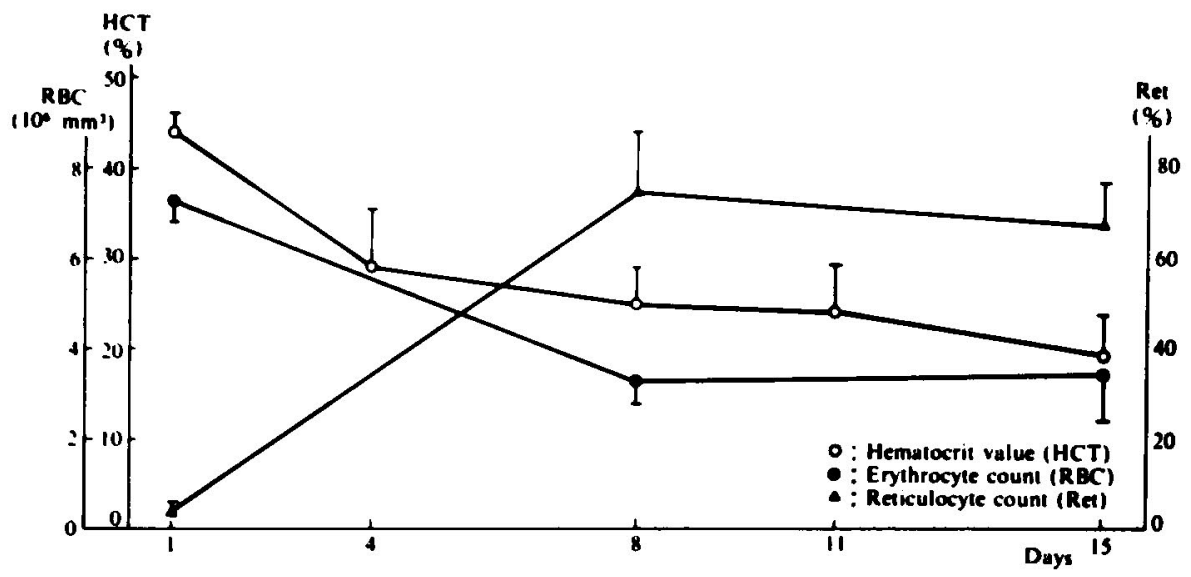

Fig. 1. The time-course of mean hematocrit value, erythrocyte and reticulocyte counts

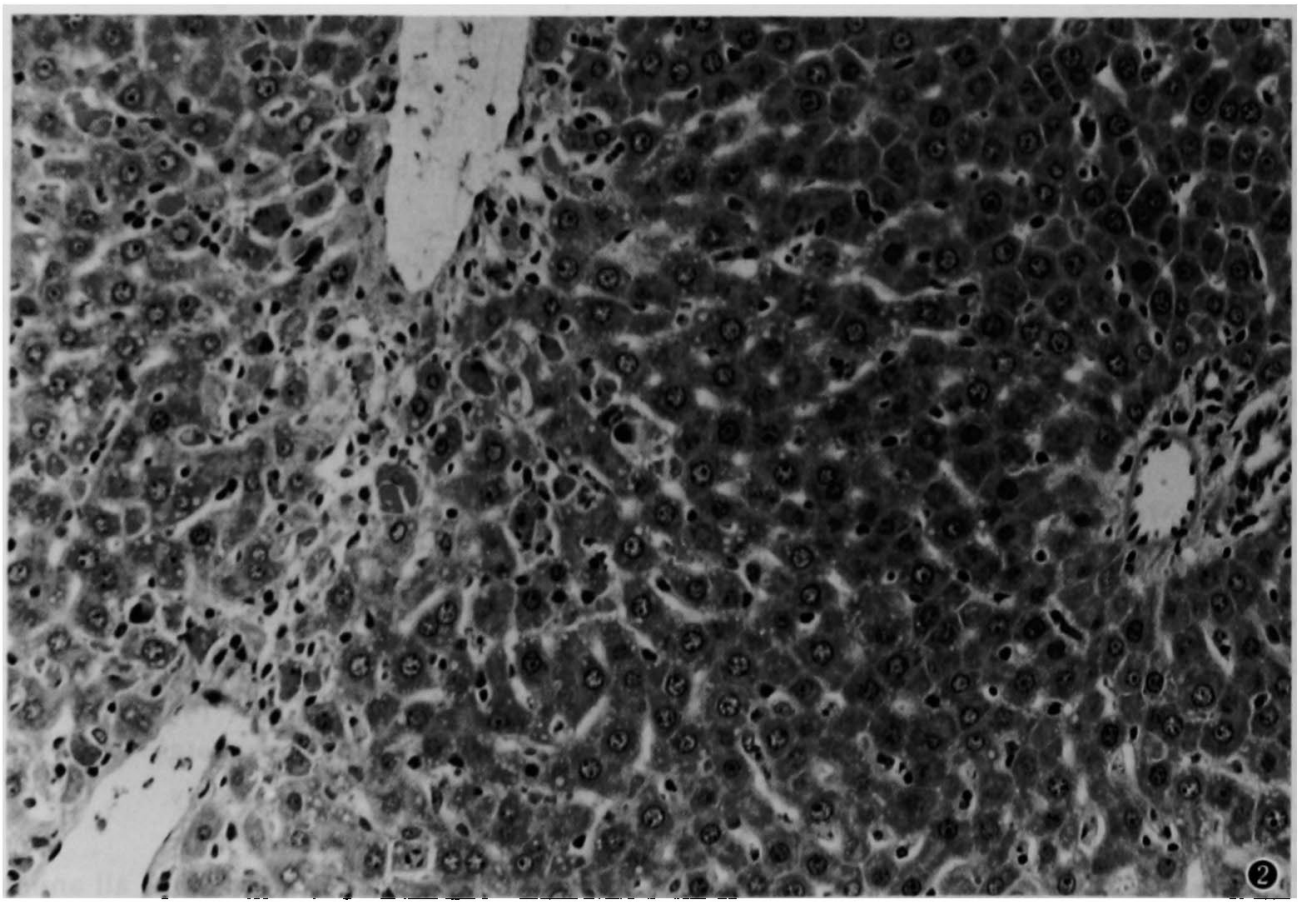

Fig. 2. Liver cell necrosis with a few acidophilic bodies was observed around the central vein. H.E., $\times 225$. 
Sakai. Yoshida, Fujimoto, $e t$ al.

under 15\%. The time-course of erythrocyte counts was similar to that of HCT values. On the other hand, reticulocyte counts increased rapidly in the first week and levelled off as plateau there- after. Mean HCT values, erythrocyte and reticulocyte counts were $19 \%, 3.4 \times 10^{6} / \mathrm{mm}^{3}$ and $67 \%$ at necropsy, respectively.

Histopathological examination revealed liver
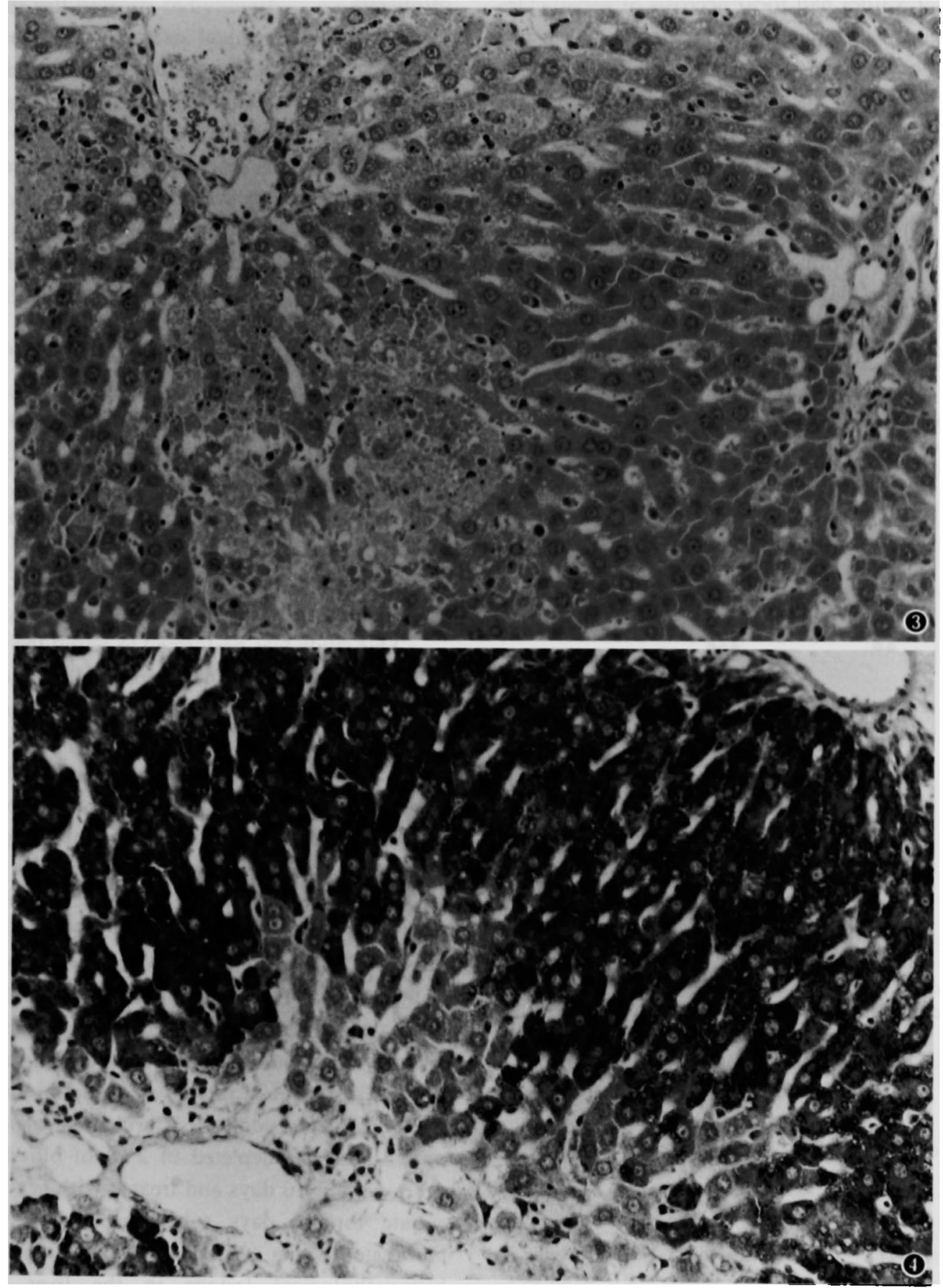

Fig. 3. Focal necrosis of liver cells. H.E., $\times 225$.

Fig. 4. No abnormality around the portal area. The glycogen preservation is normal. PAS stain, $\times 225$ 
cell necrosis around the central vein (Fig. 2) and extramedullary hematopoietic foci scattered in every lobule. Necrotic liver cells were often detached from the liver cell cord and observed as acidophilic bodies. Some animals having very low HCT values had focal necrosis of hepatocytes occasionally accompanied by infiltration of a few macrophages (Fig. 3). The liver cells in centrolobular areas had little glycogen preservation. On the other hand, there was no abnormality around the portal areas and the glycogen preservation in liver cells was normal (Fig. 4). Histological grades of liver lesion were plotted in Fig. 5 against their own HCT values. There was a tendency for hepatocellular degeneration/necrosis to be observed in the animals whose HCT value was around $20 \%$ or less. In animals showing very low HCT value, under 15\%, the grade of liver lesion was moderate to severe.

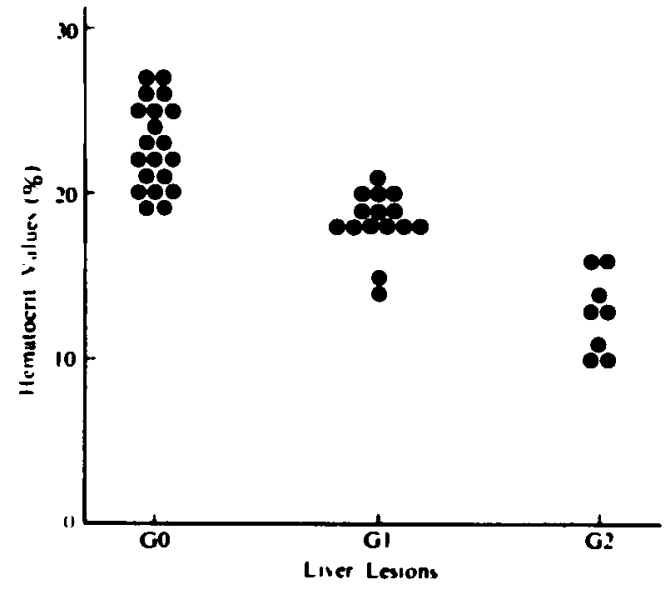

Fig. 5. Relation between liver lesions and hematocril values.

Grade 0: Normal

Grade 1: Hepatocellular degeneration/necrosis, slight

Grade 2: Hepatocellular degeneration/necrosis, moderate to severe

Table 1. Results of Plasma Biochemical Examination

\begin{tabular}{lccr}
\hline & Day 0 & Day 8 & Day 15 \\
\hline GOT (IU/1) & $87.0 \pm 12.5$ & $99.1 \pm 24.8$ & $116.0 \pm 79.7$ \\
GPT (IU/1) & $36.5 \pm 5.4$ & $42.0 \pm 9.8$ & $50.9 \pm 35.2$ \\
ALP (KAU/dl) & $90.6 \pm 24.4$ & $53.8 \pm 11.6$ & $46.6 \pm 14.6$ \\
\hline
\end{tabular}

(Mean \pm S.D.)
There were no obvious changes except for the presence of extramedullary hematopoietic foci in livers of rats whose HCT values were over $20 \%$. There was a relationship between occurrence of hepatocellular degeneration/necrosis and severity of anemia.

Plasma biochemical examination revealed a tendency for slight elevations of GOT and GPT during the study (Table 1). However, there was no clear relationship between these elevations and liver cell necrosis individually. There were no effects of anemia on ALP values.

There were no strain differences on the occurrence of liver cell necrosis and clinical parameters.

\section{Discussion}

It is well known that the liver is easy to fall into hypoxia because it is provided with blood having lower oxygen tension. Under hypoxic condition following circulatory disturbances the centrolobular zone, which is far from the portal vein and hepatic artery, may be supplied with blood of low oxygen and nutrient level. Thus, centrolobular liver cell necrosis may oocur under these conditions.

This study was performed to reveal the role of anemia on the induction of hepatocellular necrosis in rats. Rats depleted of blood from the orbital venous plexus for 2 weeks showed rapid decreases of erythrocyte counts and HCT values in the first week, and fell into a severe anemic condition. In this study, centrolobular hepatocellular degeneration/necrosis was observed in the animals whose HCT values were around $20 \%$ or less. We also reported centrolobular liver cell necrosis experimentally induced in beagle dogs by repeated phlebotomy ${ }^{\circ}$. Hayes ${ }^{8}$ reported centrolobular necrosis of hepatocytes in monkeys with hemolytic anemia, and Tamura et al..$^{10}$ observed anemia and liver cell changes in rats given an antibiotic for 5 weeks. On the contrary, Furuhama et al. ${ }^{11}$ reported that rats depleted of $2 \mathrm{ml}$ of blood every other day for 10 days and treated with gentamicin sulfate for 10 days subcutaneously developed moderate anemia (28.5\% of HCT value) but no histopathological lesions in the liver. These results suggest that severe anemic condition is necessary to induce hepatocellular necrosis experi- 
mentally.

Plasma biochemical examination revealed slight elevations of GOT and GPT levels in rats with low HCT values, but there was no clear relationship between these biochemical findings and liver cell necrosis individually. As Frederiks et al. reported", this may suggest that slight elevations or GOT or GPT cannot be used as a predictive sign of liver cell necrosis at an early stage.

In conclusion, liver cell necrosis may oocur among animals in severe anemic condition. Therefore, anemia should be considered as a causative factor of liver cell necrosis in toxicity studies in which animals are in a severe anemic condition attributable to drug administration.

\section{References}

1. Baker, HC: Ischemic necrosis in the rat liver. J Pathol Bacteriol 71 : 135-143, 1956.

2. Bassi, $M$ and Bernelli-Zazuera. A : Ultrastructural cytoplasmic changes of liver cells after reversible and irreversible ischemia. Exp Mol Pathol 3 : 332-350, 1964.

3. Chien, KR, Abrams, J, Pfau, RG, and Farber, JL Prevention by chlorpromazine of ischemic liver cell death. Am J Pathol 88 : 539-558, 1977.

4. Cohen, JA and Kaplan, MM : Left-sided heart failure presenting as hepatitis. Gastroenterology 74 : 583-587, 1978.

5. Frederiks, WM, James, J, Bosch, KS, Schrøder, MJR, and Schuyt, HC: A model for provoking ischemic necrosis in rat liver parenchyma and its quantitative analysis. Exp Pathol 22 : 245-252, 1982.

6. Frederiks, WM, Myagkaya, GL, Bosch, KS, Fronik, GM, Veen, HV, Vogels, IMC, and James, J: The value of enzyme leakage for the prediction of necrosis in liver ischemia. Histochemistry $78: 459$ 472, 1983.

7. Frederiks, WM, Fronik, GM, and Hesseling, MG : A method for quantitative analysis of the extent of necrosis in ischemic rat liver. Exp Mol Pathol 41 : 119-125, 1984

8. Hayes, KC: Pathophysiology of vitamin E deficiency in monkeys. Am J Clin Nutr 27 : 1130 1140, 1974.

9. Sakai, T, Fujimoto, R, Yoshida, S, Hanada, T, Miki, $H$, and Nomura, $Y$ : Anemia and liver cell necrosis in Beagle dogs. J Toxicol Pathol 1 : 145-148, 1988.

10. Tamura, J. Sato, N. Ezaki, H. Miyamoto, H, Oda, S, Hirai, K. Tokado, H, Matsumoto, M, and Shirai, S : Safety study on Ceftazidime (1) : acute toxicity in mouse, rat and rabbit, and subacute toxicity in rat by subcutaneous administration (in Japanese). Chemotherapy 31(S-3) : 817-841, 1983.

11. Furuhama, K, Kato, M, Suzuki, N, Igarashi, K, and Onodera, $T$. The influence of single or repeated phlebotomy on the physiological condition of normal and diseased rats. J Toxicol Sci 12 : 1-9, 1987. 